\title{
Un perfil cultural del koto, shamisen y shakuhachi ${ }^{1}$
}

DOI: $10.32870 /$ mycp.v8i25.247

Daisuke Kishi*

\section{Introducción}

$\mathrm{T}$ anto por radio como en las tiendas de cD últimamente se escucha, quizá con más frecuencia, música pop o rock, cuya corriente es seguramente norteamericana o, al menos, occidental. Esta tendencia no es exclusiva de México ni de otros países cercanos a él, sino propia de muchas partes del mundo. Por supuesto, el caso de Japón tampoco es excepcional. Sin embargo, en la tierra nipona coexisten todavía lo tradicional y lo moderno. En la arquitectura, por ejemplo en las calles del centro de Tokio, se encuentran edificios modernos y altos, equipados con alta tecnología, tiendas y cafeterías con ambiente europeo; en cambio, a unos pasos de la estación de tren llamada Harajuku, en pleno centro de la ciudad, se aprecia una entrada enorme al santuario sintoísta Meiji-jinguu, en el cual uno puede olvidarse del ruido y disfrutar, sin preocupación, de la naturaleza y la tranquilidad del bosque, así como de unas construcciones sagradas y antiguas. En la música también podremos observar algo similar. En la vida cotidiana, con la proliferación de la música escandalosa, la gente parece haberse olvidado del sonido tradicional apegado a la naturaleza, pero

* Profesor investigador del Departamento de Estudios del Pacífico de la Universidad de Guadalajara. en algunas ocasiones, como en los festivales de los diferentes barrios de las ciudades, los cuales se celebran normalmente en un santuario en la temporada de verano $\mathrm{u}$ otoño, el pueblo vuelve al pasado. Es decir, el lugar sagrado se convierte en un centro de diversiones con muchos puestos de dulces, golosinas, raspados, juegos, etc.; además, para alabar a los dioses la gente realiza la procesión conocida con el nombre de omikoshi, canta, baila y toca algunos instrumentos musicales tradicionales, tales como el koto, el shamisen, el shakuhachi, varios tipos de tambores llamados taiko, entre otros. Ahí revive la tradición japonesa, en especial en la música.

El koto es un instrumento musical o una cítara japonesa que originalmente cuenta con trece cuerdas de seda encerada. Actualmente también lo hay de diecisiete cuerdas, de seda o de nailon. Cuando se habla de música de koto, se hace referencia a que ésta puede ser la llamada soukyoku, que significa "música de koto". En general se toca sobre el suelo.

\section{Imagen del koto}

El shamisen, parecido a un banjo, es un laúd japonés con tres cuerdas, de 1.1 a 1.4 metros de largo; ${ }^{2}$ su nombre significa "cuerdas de tres sabores". Este instrumento japonés casi siempre se toca acompañado de cantos o narraciones. Existen unas variaciones que reciben el nombre de samisen 


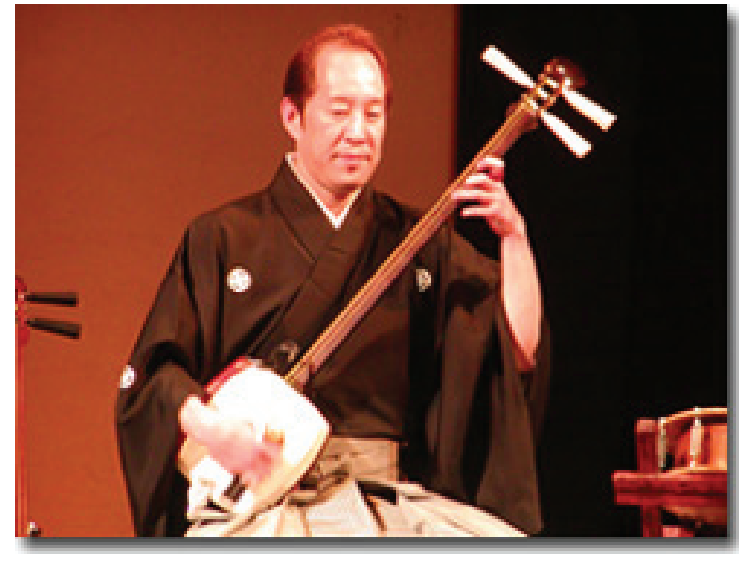

en las regiones de Kioto y Osaka, y sangen en la música cortesana. ${ }^{3}$

\section{Imagen del shamisen}

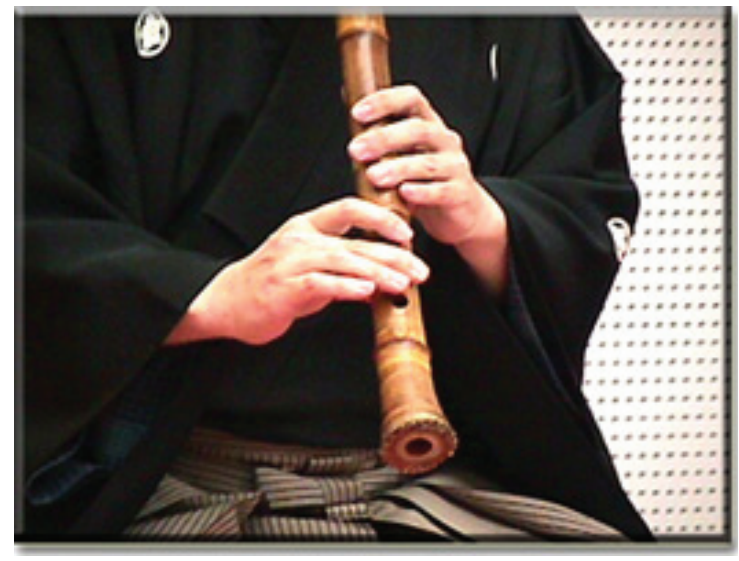

El shakuhachi es una especie de flauta hecha de bambú, con cuatro agujeros enfrente y uno atrás. Shaku es una medida que corresponde al tamaño de un pie de un japonés, y hachi significa "ocho" en japonés; es decir, "1.8 shaku" corresponde a $54.5 \mathrm{~cm}^{4}$

\section{Imagen del shakuhachi}

Estos tres instrumentos musicales parecen ser los más utilizados en la música tradicional y clásica japonesa. Han sobrevivido de forma independiente, o bien acompañados con otros instrumentos. En la actualidad se dan conciertos de los mencionados ins-

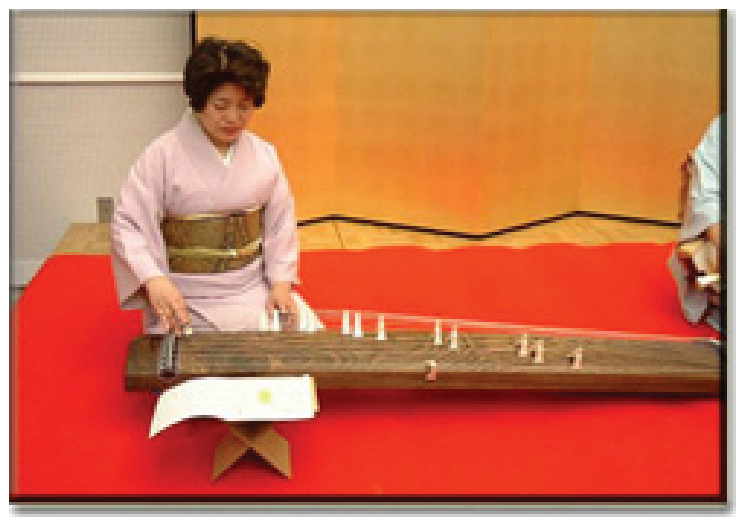

trumentos, al igual que hay una orquesta filarmónica.

\section{Origen y desarrollo histórico}

Comúnmente se cree que el koto actual data del siglo XVI, pero su verdadero origen parece estar en China; de ese país fue importado a Japón en el año 678 un instrumento de siete cuerdas llamado $k$ 'in. ${ }^{5}$ El koto más primitivo (de aquella época) es el que se conoce con el nombre de yamato-goto o wagon, de cinco cuerdas. Luego empezó a usarse con seis cuerdas en la Era de Nara (710-794), en la cual apareció también el koto de trece cuerdas basado en el instrumento chino cheng. En este sentido, es decir, en el sentido del número de cuerdas, el origen del koto moderno quizá esté en esa época.

Inicialmente, en el siglo viII, el koto, acompañado de otros instrumentos, tales como tambores, pequeño gong; shakuha$c h i^{6}$ y laúd de cuatro cuerdas, se usaba para la música cortesana conocida con el nombre de gagaku (que significa literalmente "música elegante"). Esta música se puede oír también hoy en día en algunas ceremonias de los santuarios sintoístas. De hecho, en el siglo XVI un sacerdote budista (Kenjun) compuso unas canciones acompañadas del koto, cuya música normalmente se considera como verdadero género de la de koto. En otras palabras, puede ser el 
inicio de la música de koto oficialmente. En esa época apareció también la música con otro tipo de instrumento llamado shamisen, que es una especie de mandolina de tres cuerdas. Estos dos instrumentos se fueron combinando poco a poco. Este estilo se llama te-goto, y fue desarrollado principalmente en Kioto y Osaka.

El shamisen tampoco parece ser originario de Japón. Según Kishibe, ${ }^{7}$ en el siglo XIII se usaba en China un instrumento con tres cuerdas llamado san-hsien, el cual llegó primero a las islas de Ryukyu —que es actualmente la prefectura de Okinawa-, y en 1562 a la tierra nipona. Al principio lo tocaban los cantantes ambulantes y las geishas. Cuando se hizo popular dicho instrumento fue en la Era de Edo (1600-1868). Está fuertemente vinculado con la vida ociosa de la gente, así como con actividades teatrales como el kabuki y el bunraku. Es muy rara, de hecho, la interpretación de la música del shamisen sin acompañamiento de cantos o narraciones, excepto en el caso de Tsugaru-jamisen, el cual fue desarrollado en la región de Tsugaru, prefectura de Aomori.

El shakuhachi, también originario de China, en la Era de Nara (710-793), se usaba en la música cortesana, pero durante mucho tiempo no había habido repercusión. La mencionada flauta japonesa moderna probablemente proviene de la que usaban a principios de la Era de Edo algunos monjes budistas, en especial del budismo Zen. Estos monjes buscaban, por medio de la interpretación del shakuhachi, el control de la respiración y la emisión del sonido áspero para purificarse. ${ }^{8}$ En este sentido, el son de dicha flauta japonesa, al menos originalmente, tenía un valor más espiritual o religioso. Desde el punto de vista musical, Kinko Kurosawa (1710-1771) estableció un estilo artístico de dicha música sobre la base del shakuhachi Fuke. ${ }^{9}$

En la misma época, Kengyo Yamada (1757-1817) estableció en Edo (Tokio actual) una corriente nueva para la música del koto, introduciendo la música del shamisen en la del koto. En realidad, en ese periodo su música se hizo más popular, ya que apareció otro tipo de música del koto en conjunto: koto, shamisen y shakuhachi; este conjunto musical se conoce con el nombre de sankyoku ("tres piezas").

Al principio de la Era de Meiji (18681912) empezó una occidentalización. En el campo de música kotista, Michio Miyagi (1895-1956) fue el primer compositor que combinó la música de koto con la europea, por ejemplo, con el estilo orquestal. Creemos que mucha música actual en que se usa el koto está basada en su estilo. Podemos citar algunos grupos que lo están manejando. El primer grupo que se presenta se llama Kasou Kai (Asociación de Amigos de la Música Japonesa), que es un cuarteto mexicano-japonés. Ellos interpretan la música de koto, acompañado de una flauta o violín, e incluso de un piano. Otro grupo japonés de este estilo sería Rin que está formado por tres chicas. ${ }^{10}$ Interpretan canciones tocando ellas mismas el koto, el shakuhachi y el biwa, el cual se toca como guitarra o shamisen con un tono un poco nostálgico. Sus interpretaciones parecen considerarse más modernas, puesto que el género posiblemente puede pertenecer a la música pop, pero con un matiz tradicional de Japón, en especial por el acompañamiento de estos instrumentos clásicos.

\section{Estructura física}

La cítara koto mide $186 \mathrm{~cm}$ de largo y 48 cm de ancho, normalmente está hecha de madera de paulonia porque ésta tiene una 
calidad suave y adecuada para las cuerdas de seda. El koto tradicional lleva trece cuerdas de seda con el mismo grueso. Para la afinación de cada cuerda se utilizan unos puentecitos con una distancia diferente de uno a otro, es decir, las notas dependen de dónde se sitúe el puentecito.

Actualmente muchos músicos de koto o kotistas prefieren el uso de dicho instrumento con diecisiete cuerdas, pues según los especialistas produce un sonido más profundo. Este modelo lo inventó el gran maestro Michio Miyagi en la Era de Meiji que acabamos de mencionar. Martínez R. afirma que existen más variedades del koto: con 23 cuerdas, 30 cuerdas e incluso uno de 80 cuerdas que cuenta con las sonoridades y la capacidad del piano. ${ }^{11}$

Los kotistas lo tocan con las púas puestas en el dedo pulgar, índice y medio de la mano derecha. Usan la mano izquierda para recolocar durante la interpretación los puentecitos en dependencia de la necesidad. Existen dos escuelas importantes, entre otras. En la escuela Ikuta usan púas más delgadas y cuadradas, mientras que en la de Yamada las usan más gruesas y redondas. Y esta diferencia morfológica produce cierta variación en tono o timbre.

Como hemos visto, el shamisen es una especie de banjo de cuello largo con tres cuerdas. Lo más distintivo de este instrumento es el uso de piel de gato. Y en algunas ocasiones también se usa la de perro, aunque es menos común y menos elegante. Se toca normalmente con un plectro de hueso. Es interesante mencionar que en Okinawa se fabrican con piel de víbora desde la llegada de dicho instrumento de China, ya que el san-hsien chino está cubierto de piel de víbora; además en la región okinawaense abundan las víboras, tal vez por su clima y naturaleza. El shamisen, a diferencia del koto, puede ser muy portátil, puesto que se puede separar en tres partes con facilidad.

Para el shakuhachi normalmente se utiliza la parte cercana de la raíz del bambú, por eso se observa que la forma del aparato no es muy estilizada, sino que da una impresión un poco rústica. Esta característica parece reflejar la vida de los monjes budistas. El diámetro de la mencionada flauta japonesa, por ser utilizado el bambú, es de 4 a $5 \mathrm{~cm}$. Como es sabido por los músicos y fanáticos, la digitación sutil de los cinco orificios, la presión del aire y el cambio de ángulo de dicho instrumento hacen que el sonido sea variable y misterioso.

\section{Los tres instrumentos musicales tradicionales dentro de la sociedad japonesa}

Como hemos visto, la música de koto antiguamente era religiosa o de la clase alta, ya que se interpretaba algunas veces en los santuarios sintoístas y otras en las cortes. Realmente, la música de koto se sigue considerando elegante. Hasta hace poco saber tocar ese instrumento había sido uno de los requisitos para que las mujeres se casaran de manera elegante, cuya costumbre data al menos de la época de Meiji, según afirma Bellessort, ${ }^{12}$ pues se creía que las mujeres educadas deberían tener conocimiento profundo y práctico de la cultura japonesa, tal como de la ceremonia de té, del arreglo de flores o Ikebana, o saber tocar algún instrumento musical tradicional para realizar esas presentaciones culturales en casa en un momento dado. Sin embargo, últimamente esta costumbre se ha ido perdiendo en la sociedad nipona, y se considera más bien como un hobby o pasatiempo de buen gusto y de categoría.

El shamisen abrió su camino en la época de Edo, junto con el teatro Kabuki y 
el Bunraku, el de títeres. En el Kabuki el shamisen acompaña a los cantos durante la actuación de los actores, mientras que en el Bunraku las narraciones son la pareja de dicho instrumento. En este aspecto, el shamisen ha sido quizá uno de los instrumentos musicales más apegados al teatro nipón. En comparación con la difusión más amplia del koto entre las mujeres, tal vez por su sonido elegante y fino, parece haber afición al shamisen independientemente del sexo, aunque sea el instrumento musical preferido por las geishas. De hecho, las geishas están representadas por mujeres vestidas de kimono que saben tocar instrumentos musicales, cantar, bailar y actuar como edecanes, pero con sabiduría e inteligencia. En su campo también existe servicio a domicilio; es decir, por la solicitud de los clientes

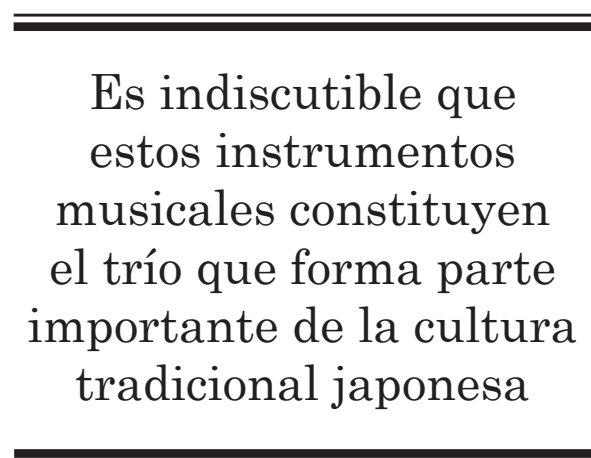

ellas van al hotel o al restaurante donde hay salones de fiesta. El instrumento musical que llevan casi sin excepción puede ser el llamado shamisen, sea por su función artística, sea por su portabilidad.

El shakuhachi probablemente es uno de los instrumentos musicales que más han servido de medio de meditación o de relajamiento. Esto se debe indudablemente a su sonido más apegado a la naturaleza, cuya sensación es quizá el viento que corre por el bosque de bambúes. Con razón lo preferían usar los monjes budistas en aquella época. Sin embargo, actualmente hay algunos shakuhachistas que tocan como una flauta piezas musicales europeas, pero el sonido no deja de ser japonés o asiático.

A pesar de la historia y las características propias de cada instrumento, es muy filosófico. común encontrar en un concierto estos tres instrumentos en conjunto, cuyos sonidos parecen complementarse.

\section{Conclusión}

Como hemos visto, el instrumento de koto, está hecho de madera de paulonia, que es la más apropiada para el clima de Japón, y de cuerdas de seda, la cual es un símbolo de elegancia tanto en Japón como en muchos países, por tal razón podremos decir que es uno de los instrumentos musicales más representativos del país. El tono del koto japonés, si lo escuchamos detenidamente, refleja su cultura misma. En otros términos, el sonido del koto se oye mejor en armonía con la naturaleza, nos da una sensación pacífica y elegante. Quizá por eso es muy común escuchar en Japón esa música en el Año Nuevo, fecha importante para los japoneses tanto espiritual como simbólicamente.

Desde el punto de vista social el shamisen, a diferencia del koto, que ha tenido más aceptación por la clase alta y por las mujeres, es el que se ha difundido a través de todas las clases sociales y de ambos sexos. Por otra parte, este instrumento de tres cuerdas quizá tenga un valor más artístico por su fuerte vinculación con el teatro. El shakuhachi, por su origen, generalmente es favorecido por los hombres; podrá considerarse más religioso o

Por último no debemos dejar de tomar en cuenta el origen común de los mencionados instrumentos musicales, ya que son todos originarios de China, pero con 
modificaciones y adaptaciones sutiles a la sociedad y la naturaleza niponas. Esto puede coincidir curiosamente con el comportamiento de los japoneses en particular en el campo de tecnología. Así, pues, parece que los japoneses tenemos una capacidad talentosa de imitar lo existente y mejorar su calidad. De todos modos, es indiscutible que estos instrumentos musicales constituyen el trío que forma parte importante de la cultura tradicional japonesa. mo

\section{Notas}

1 Este artículo se ha basado parcialmente en una conferencia que di el día 26 de agosto de 2005 en el CUAAD de la Universidad de Guadalajara, con el título: "Introducción al sonido del koto".

2 Japan: An Illustrated Enciclopedia. 2., Tokio, Kodansha, 1993, p. 1356.

3 Ibídem.
4 Kishibe, Shigeo. The tradicional Music of Japan. Tokio, Ongaku no Tomo Sha, 1984, pp.78-79.

5 Confucio fue maestro de este instrumento; aprender a tocarlo se consideraba un elemento importante para formar una buena educación, con motivo de enriquecer el corazón y el espíritu.

6 Esta flauta asiática apareció en Japón a finales del siglo VII (Japan: An Illustrated Enciclopedia. 2. Tokio, Kodansha, 1993, p. 1355), cuya etapa coincide totalmente con la de la llegada del koto.

7 Kishibe, S., op. cit. pp. 69-74.

8 Véase www.rutadeseda.org/Japon/JPShakuhachi.html.

9 Es el que empleaban los monjes budistas llamados "komusou" de la secta Fuke, quienes trabajaban con los samuráis (Kishibe, S. op. cit. p. 79).

10 Se pueden escuchar algunas de sus obras en la página web: www.avexnet.or.jp/rin.

11 Martínez R., Mauricio. www.rutadeseda.org/Japon/JPKoto.html.

12 Bellessort, Andrés. La sociedad japonesa. Barcelona, Montaner y Simón, 1905, pp. 237-242. 